\title{
Generalized biorthogonal Daubechies wavelets
}

\author{
Cédric Vonesch, Thierry Blu and Michael Unser \\ Biomedical Imaging Group, Ecole Polytechnique Fédérale de Lausanne, Switzerland
}

\begin{abstract}
We propose a generalization of the Cohen-Daubechies-Feauveau (CDF) and 9/7 biorthogonal wavelet families. This is done within the framework of non-stationary multiresolution analysis, which involves a sequence of embedded approximation spaces generated by scaling functions that are not necessarily dilates of one another. We consider a dual pair of such multiresolutions, where the scaling functions at a given scale are mutually biorthogonal with respect to translation. Also, they must have the shortest-possible support while reproducing a given set of exponential polynomials. This constitutes a generalization of the standard polynomial reproduction property.

The corresponding refinement filters are derived from the ones that were studied by Dyn et al. in the framework of non-stationary subdivision schemes. By using different factorizations of these filters, we obtain a general family of compactly supported dual wavelet bases of $L_{2}$. In particular, if the exponential parameters are all zero, one retrieves the standard CDF B-spline wavelets and the $9 / 7$ wavelets. Our generalized description yields equivalent constructions for E-spline wavelets.

A fast filterbank implementation of the corresponding wavelet transform follows naturally; it is similar to Mallat's algorithm, except that the filters are now scale-dependent. This new scheme offers high flexibility and is tunable to the spectral characteristics of a wide class of signals. In particular, it is possible to obtain symmetric basis functions that are well-suited for image processing.
\end{abstract}

Keywords: Biorthogonal, wavelet, non-stationary, mutiresolution, reproduction, exponential polynomials

\section{INTRODUCTION}

In a previous paper, ${ }^{1}$ we focused on non-stationary multiresolution spaces generated by a set of orthonormal and compactly supported scaling functions that can reproduce a given set of exponential polynomials. These represented a natural extension of the orthonormal Daubechies wavelets. While using essentially the same computational structure as conventional wavelets (perfect reconstruction filter banks), they can be adapted to the analysis of specific types of signals. For instance, narrow-band signals can accurately be approximated by polynomials modulated at the suitable frequency. However, the design of orthonormal wavelets requires a step known as spectral factorization, which can make the filter lengths grow when going to coarser scales; moreover, orthogonal filters cannot be symmetric. These limitations are also encountered in classical wavelet filter design, and they can be circumvented by relaxing the orthogonality condition and considering biorthogonal structures.

Here, we extend our construction to dual multiresolution analyses that contain a given set of exponential polynomials. After a brief description of such structures in Sect. 2, we introduce the corresponding filter-bank decomposition in Sect. 3. In Sect. 4, we relate the reproduction of exponential polynomials to the roots of the filters. We further characterize these filters in Sect. 5, which leads to an extension of two families of biorthogonal wavelets, commonly referred to as Cohen-Daubechies-Feauveau and 9/7 wavelets (Sect. 6).

\section{NON-STATIONARY MULTIRESOLUTIONS}

\subsection{Definition}

The fundamental structure that underlies our construction is a set of embedded, shift-invariant approximation spaces. The important difference with stationary multiresolutions encountered in classical wavelet theory is that each space is generated using a different function, ${ }^{2}$ which depends on the scale parameter $j$. In addition, we impose that these so-called scaling functions have integer translates that are linearly independent. 
Definition 2.1 (Non-Stationary multiresolution). Given the scaling functions $\left(\varphi_{j}\right)_{j \in \mathbb{Z}}$, the spaces

$$
V_{j}=\operatorname{span}\left\{\varphi_{j}\left(\frac{t-2^{j} k}{2^{j}}\right), k \in \mathbb{Z}\right\}
$$

define a non-stationary multiresolution if and only if

- for any $j \in \mathbb{Z},\left(\varphi_{j}\left(t / 2^{j}-k\right)\right)_{k \in \mathbb{Z}}$ is a Riesz basis of $V_{j}$;

- for any $j \in \mathbb{Z}, V_{j+1} \subset V_{j}$;

- $\bigcup_{j \in \mathbb{Z}} V_{j}$ is dense in $L^{2}(\mathbb{R})$;

- $\bigcap_{j \in \mathbb{Z}} V_{j}=\{0\}$.

\subsection{Basic Properties}

The embedding of the spaces $V_{j}$ implies the existence of scaling filters $h_{j}[k] \in \ell^{2}(\mathbb{Z})$ such that

$$
\varphi_{j+1}\left(\frac{t}{2^{j+1}}\right)=\sum_{k \in \mathbb{Z}} h_{j}[k] \varphi_{j}\left(\frac{t-2^{j} k}{2^{j}}\right) .
$$

Conversely this scaling relation implies that any function in $V_{j+1}$ can be expressed as a linear combination of the basis functions of $V_{j}$, hence providing a necessary and sufficient condition for $V_{j+1} \subset V_{j}$.

In the Fourier-domain, the scaling relation (2) reads

$$
2 \hat{\varphi}_{j+1}(2 \omega)=H_{j}\left(e^{i \omega}\right) \hat{\varphi}_{j}(\omega) .
$$

A consequence of this relation is the infinite-product formula, which defines the scaling functions using the scaling filters only, provided $\lim _{j \rightarrow-\infty} \hat{\varphi}_{j}\left(2^{j} \omega\right)=1$. In this case

$$
\hat{\varphi}_{j}(\omega)=\prod_{\ell=1}^{+\infty} \frac{1}{2} H_{j-\ell}\left(e^{i 2^{-\ell} \omega}\right) .
$$

\section{BIORTHOGONAL MULTIRESOLUTION DECOMPOSITION}

In what follows, we are concerned with pairs of multiresolution analyses $\left(\tilde{V}_{j}\right)_{j \in \mathbb{Z}},\left(V_{j}\right)_{j \in \mathbb{Z}}$ (resp. the "analysis" and "synthesis" spaces) that provide a practical structure for multiscale signal approximation. This is achieved by enforcing a biorthogonality relation between the integer shifts of their respective scaling functions: at any scale $j \in \mathbb{Z}$,

$$
\left\langle\tilde{\varphi}_{j}, \varphi_{j}(\cdot-k)\right\rangle=\delta[k] .
$$

To approximate a function $f \in L^{2}(\mathbb{R})$, we can then use

$$
\tilde{P}_{V_{j}} f(t)=\sum_{k \in \mathbb{Z}} x_{j}[k] 2^{-j / 2} \varphi_{j}\left(t / 2^{j}-k\right),
$$

where $x_{j}[k]=\left\langle f, 2^{-j / 2} \tilde{\varphi}_{j}\left(\cdot / 2^{j}-k\right)\right\rangle$, which defines a projection operator on $V_{j}$ (perpendicularly to $\left.\tilde{V}_{j}\right)$, at each scale $j$.

This continuous-time approximation framework is related to discrete filter-bank structures through the following simple property. Notice that all scaling functions (and thus the filters) are assumed to be real.

Property 1 (Biorthogonal Filters). If the biorthogonality relation (5) holds for any $j \in \mathbb{Z}$, then the $z$-transforms of the scaling filters $h_{j}[k]$ and $\tilde{h}_{j}[k]$ must satisfy

$$
H_{j}(z) \tilde{H}_{j}\left(z^{-1}\right)+H_{j}(-z) \tilde{H}_{j}\left(-z^{-1}\right)=4
$$




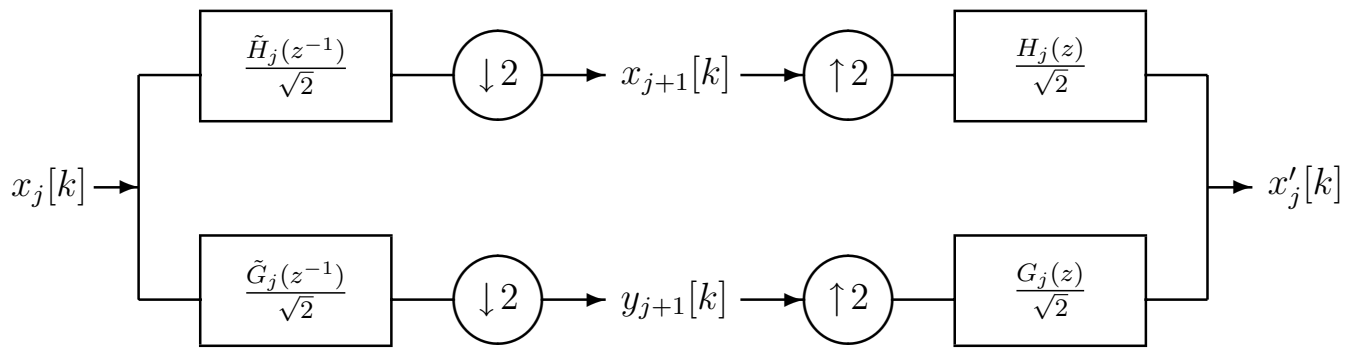

Figure 1. Non-stationary filter bank.

for all $j$.

Equivalently, (7) states that the discrete filters $h_{j}[k] / \sqrt{2}$ and $\tilde{h}_{j}[k] / \sqrt{2}$ are biorthogonal with respect to their even translates. For the filter bank in Fig. 1 this ensures perfect reconstruction (i.e. $x_{j}^{\prime}[k]=x_{j}[k]$ ), provided that one sets $G_{j}(z)=z^{n} \tilde{H}_{j}\left(-z^{-1}\right)$ and $\tilde{G}_{j}(z)=z^{n} H_{j}\left(-z^{-1}\right)$, where $\mathrm{n}$ is an odd integer.

This structure also describes an efficient way to compute coarser approximations of a function $f$ from a given projection $\tilde{P}_{V_{j}} f$. More precisely, if $x_{j}[k]$ denotes the components of the projection at scale $j$ (see $(6)$ ), then $x_{j+1}[k]$, which represents $\tilde{P}_{V_{j+1}} f$, is obtained from the upper branch of the filter bank in Fig. 1.

Similarly, we may introduce the wavelet functions

$$
\psi_{j}(t)=\sum_{k \in \mathbb{Z}} g_{j-1}[k] \varphi_{j-1}(2 t-k),
$$

and use them to generate the spaces

$$
W_{j}=\operatorname{span}\left\{\psi_{j}\left(\frac{t-2^{j} k}{2^{j}}\right), k \in \mathbb{Z}\right\} .
$$

Then the biorthogonality relation (7) and the definition of the filters $g_{j}[k]$ and $\tilde{g}_{j}[k]$ imply that $V_{j}=V_{j+1} \oplus W_{j+1}$

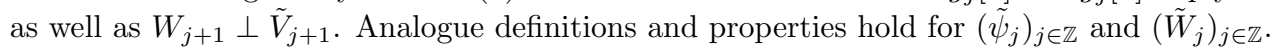

We may also define the projection operator

$$
\tilde{P}_{W_{j}} f(t)=\sum_{k \in \mathbb{Z}} y_{j}[k] 2^{-j / 2} \psi_{j}\left(t / 2^{j}-k\right),
$$

where $y_{j}[k]=\left\langle f, 2^{-j / 2} \tilde{\psi}_{j}\left(\cdot / 2^{j}-k\right)\right\rangle$. Then the second channel of the filter bank in Fig. 1 computes the components $y_{j+1}[k]$ from $x_{j}[k]$. The perfect reconstruction property ensures that $\tilde{P}_{V_{j}} f=\tilde{P}_{V_{j+1}} f+\tilde{P}_{W_{j+1}} f$.

Notice that the semi-orthogonal construction of Khalidov et al. ${ }^{3}$ also leads to this type of nonstationary multiresolution pairs, except that $\tilde{V}_{j}=V_{j}$ and $\tilde{W}_{j}=W_{j}$; this typically implies that some of the scaling functions and wavelets are infinitely supported. Here, we will constrain the scaling functions (and thus the wavelets) to be compactly supported both on the analysis and the synthesis side. This implies that the scaling filters must be FIR, allowing a simple and numerically efficient filter-bank implementation that is strictly equivalent to Mallat's Fast Wavelet Transform. Since the involved filters depend on the scale $j$, the only adaptation needed is to precompute them up to the desired coarseness level. 


\section{REPRODUCTION OF EXPONENTIAL POLYNOMIALS}

We shall now discuss the conditions under which all the approximation spaces $V_{j}$ can contain a given set of exponential polynomials.

THEOREM 4.1. Assume that the biorthogonality relation (7) holds and that the scaling functions $\left(\varphi_{j}\right)_{j \in \mathbb{Z}}$ are compactly supported and such that $\lim _{j \rightarrow-\infty} \hat{\varphi}_{j}\left(2^{j} \omega\right)=1$. Then the spaces $\left(V_{j}\right)_{j \in \mathbb{Z}}$ contain the exponential polynomials $P(t) e^{\alpha t}, \operatorname{deg} P(t) \leq N-1$ if and only if each filter $H_{j}(z)$ has a zero of order $N$ at $z=-e^{2^{j} \alpha}$, for any $j \in \mathbb{Z}$.

This result can be extended to handle the case where we would like the spaces $V_{j}$ to contain exponential polynomials with different parameters. To formalize this, we consider a vector $\vec{\alpha} \in \mathbb{C}^{N}$ and we denote $\left(\alpha_{(m)}\right)_{m \in\left[1, N_{d}\right]}$ its distinct components of respective multiplicities $N_{(m)}$. Then the spaces $V_{j}$ contain the functions $P(t) e^{\alpha_{(m)} t}$, $\operatorname{deg} P(t) \leq N_{(m)}-1$ if and only if each filter $H_{j}(z)$ is divisible by $R_{2^{j} \vec{\alpha}}(z)$, where

$$
R_{\vec{\alpha}}(z)=\prod_{n=1}^{N}\left(1+e^{\alpha_{n}} z^{-1}\right) .
$$

\section{GENERALIZED DESLAURIERS-DUBUC INTERPOLATORS}

In order to find a family of solutions of (7), we first consider the auxiliary equation

$$
H_{j}(z)+H_{j}(-z)=2
$$

where $H_{j}(z)$ is of the form $R_{2^{j}} \vec{\alpha}(z) Q_{j}(z)$, following Th. 4.1. This type of filters has recently been studied by Dyn et al. ${ }^{4}$ in the framework of generalized Deslauriers-Dubuc interpolatory subdivision schemes. ${ }^{5}$ Here, we further require that $H_{j}(z)$ is symmetric. Since it should also be real, this means that if $\alpha \in \mathbb{C}$ is an element of $\vec{\alpha}$, then so must $\alpha^{*},-\alpha$ and $-\alpha^{*}$, with the same multiplicity.

We aim at determining the shortest possible filter $Q_{j}(z)$ such that (12) holds. With this in mind, it is possible to perform the standard change of variable $Z=\left(z+z^{-1}\right) / 2$ and to get polynomials $A(Z), B(Z) \in \mathbb{R}[Z]$ such that

$$
\left\{\begin{aligned}
R_{2^{j} \vec{\alpha}}(z) & =A\left(\frac{z+z^{-1}}{2}\right) \\
Q_{j}(z) & =B\left(\frac{z+z^{-1}}{2}\right)
\end{aligned}\right.
$$

The existence of a solution to (12) is then ensured by the following mathematical result:

Theorem 5.1 (BÉzout). Given $A(Z) \in \mathbb{R}[Z]$, there exists a polynomial $B(Z) \in \mathbb{R}[Z]$ such that

$$
A(Z) B(Z)+A(-Z) B(-Z)=4
$$

if and only if $A(Z)$ has no pair of opposite roots. In this case there exists a unique polynomial $B_{0}(Z) \in \mathbb{R}[Z]$ satisfying (14) and such that $\operatorname{deg} B_{0}(Z)<\operatorname{deg} A(Z)$.

Notice that one also encounters the Bézout equation (14) in the orthonormal case. ${ }^{1}$

\section{GENERALIZED BIORTHOGONAL WAVELETS}

\subsection{Generalized Cohen-Daubechies-Feauveau Wavelets}

From the previous section, one can easily derive a generalization of the family of biorthogonal B-spline wavelets described by Cohen, Daubechies and Feauveau. ${ }^{6}$ The procedure essentially consists in taking certain roots from $R_{2^{j} \vec{\alpha}}(z)$ (that were part of $H_{j}(z)$ in Equation (12)) and moving them to $\tilde{H}_{j}\left(z^{-1}\right)$, so as to obtain solutions of $(7)$. Then

It is therefore useful to introduce vectors $\vec{\gamma}$ and $\overrightarrow{\tilde{\gamma}}$ such that $\vec{\alpha}=(\vec{\gamma}: \overrightarrow{\tilde{\gamma}})$, where (:) denotes concatenation.

$$
A\left(\frac{z+z^{-1}}{2}\right)=R_{2^{j} \vec{\gamma}}(z) R_{2^{j} \vec{\gamma}}(z) .
$$




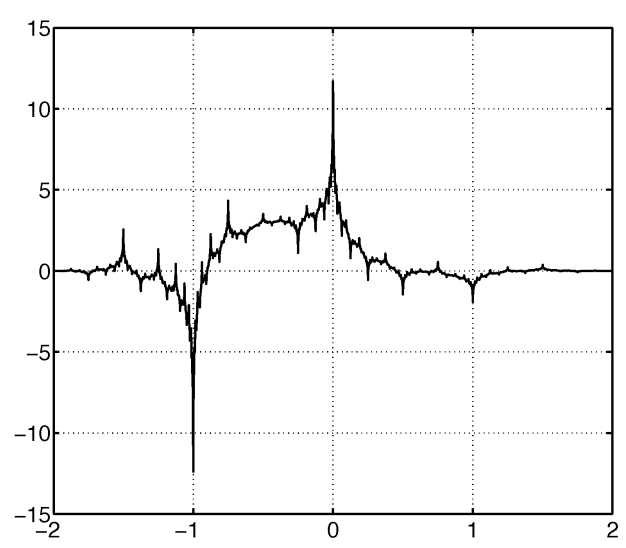

(a)

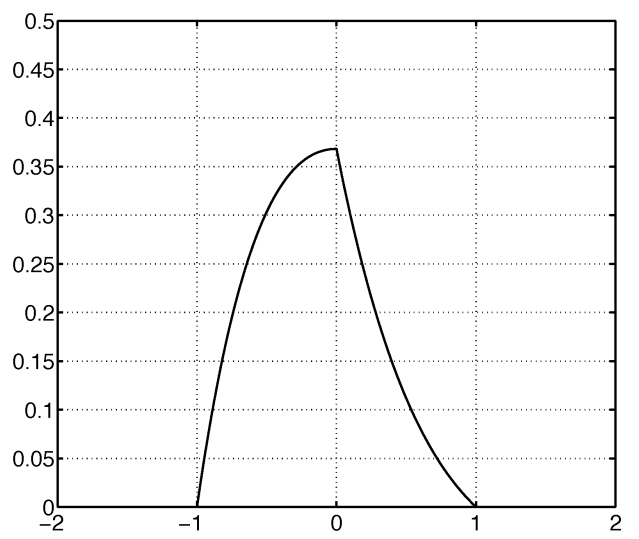

(b)

Figure 2. Generalized Cohen-Daubechies-Feauveau scaling functions $\tilde{\varphi}_{0}(t)$ (left) and $\varphi_{0}(t)$ (right), obtained for $\vec{\alpha}=$ $[1,1,-1,-1]$ and $\vec{\gamma}=[-1,-1]$. Both functions reproduce exponential polynomials of the form $P(t) e^{-t}, \operatorname{deg} P(t) \leq 1$.

Since we are looking for real filters, one must pay attention to the fact that distinct complex-conjugate elements of $\vec{\gamma}$ (and a fortiori $\overrightarrow{\tilde{\gamma}}$ ) must have the same multiplicity. Provided this condition is satisfied, one can freely choose $\vec{\gamma}$ and $\overrightarrow{\tilde{\gamma}}$ and set:

$$
\left\{\begin{array}{l}
H_{j}(z)=R_{2^{j} \vec{\gamma}}(z) \\
\tilde{H}_{j}(z)=R_{2^{j} \vec{\gamma}}\left(z^{-1}\right) Q_{j}(z)
\end{array}\right.
$$

where $Q_{j}(z)$ is obtained from the lowest-degree Bézout solution $B_{0}(Z)$. Figure 2 shows an example of the corresponding functions at scale $j=0$. It should be noted that the synthesis scaling function $\varphi_{0}(t)$ is an exponential B-spline of parameter $\vec{\gamma}_{\cdot}{ }^{7}$ In particular, it is possible to design symmetric scaling functions and wavelets by adequately choosing the $\vec{\gamma}$ parameter, which can be of interest for image processing. The classical construction is retrieved by setting $\vec{\alpha}=\overrightarrow{0}$; then $\varphi_{0}(t)$ is a conventional B-spline.

It should be mentioned that, as in the classical framework, not all combinations for $\vec{\gamma}$ and $\overrightarrow{\tilde{\gamma}}$ guarantee that the infinite product formula $(4)$ for $\tilde{\varphi}_{j}(t)$ will converge to a regular function.

\subsection{Generalized $9 / 7$ Wavelets}

Additionally, it is also possible to split $B_{0}\left(\left(z+z^{-1}\right) / 2\right)$ into individual factors and to affect them to either $H_{j}(z)$ or $\tilde{H}_{j}\left(z^{-1}\right)$. If we further impose that the resulting filters should be symmetric, we can operate directly on $B(Z)$. In particular, this can lead to a generalization of the so-called " $9 / 7$ " filters used for image coding. ${ }^{8}$

As an example, we consider $\vec{\gamma}=\overrightarrow{\tilde{\gamma}}=[2 i \pi / 5,2 i \pi / 5,-2 i \pi / 5,-2 i \pi / 5]$ and thus $\vec{\alpha}=\vec{\gamma}: \overrightarrow{\tilde{\gamma}} \in \mathbb{C}^{8}$. In this case $B_{0}(Z) \in \mathbb{R}[Z]$ is of degree 3 and has 2 complex-conjugate roots. Consequently, there is only one non-trivial factorization leading to real-valued filters. The corresponding scaling functions are plotted in Fig. 3 .

\section{CONCLUSION}

The examples presented illustrate the flexibility of the proposed design method. Most importantly, it is possible to incorporate a priori knowledge on the characteristics of the signals to be analysed into the approximation spaces, via the exponential parameters. Then one has the choice between biorthogonal E-spline wavelets or "almost orthonormal" 9/7 wavelets. Both cases allow for symmetric functions. 


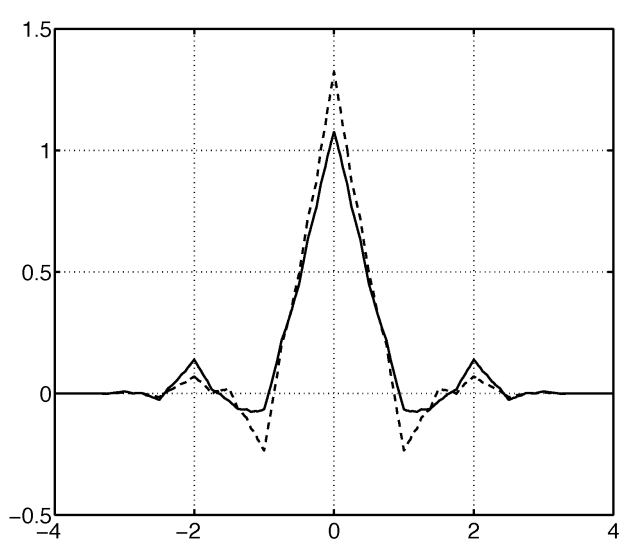

(a)

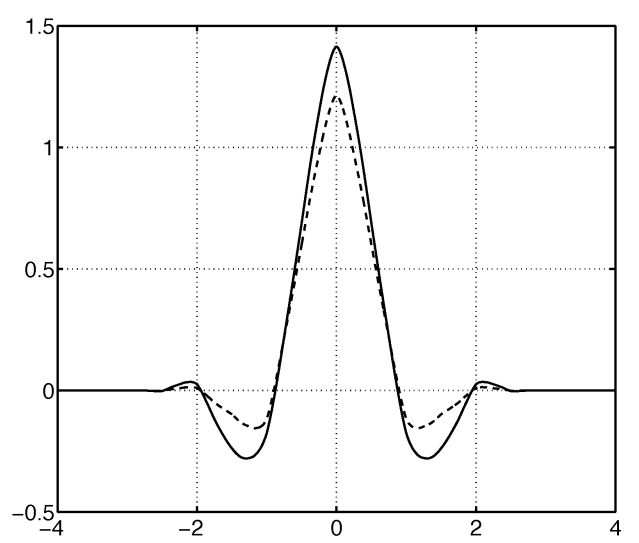

(b)

Figure 3. Biorthogonal scaling functions $\tilde{\varphi}_{0}(t)$ (left) and $\varphi_{0}(t)$ (right) corresponding to a generalization of the $9 / 7$ filters (see text). The functions obtained from the classical $9 / 7$ filters are represented using dashed lines.

Compared to the previously studied orthonormal case, this makes the corresponding filters attractive for new tasks, such as image processing (e.g. texture analysis). In general, for a given class of signals with significant harmonic or exponential trends (e.g. speech, OCT or neuronal signals), the proposed multiresolution structures may provide sparse representations. This can be useful in a number of applications, such as coding, detection or denoising.

\section{REFERENCES}

1. C. Vonesch, T. Blu, and M. Unser, "Generalized Daubechies wavelets," Proceedings of the International Conference on Acoustics, Speech and Signal Processing, 2005.

2. C. de Boor, R. DeVore, and A. Ron, "On the construction of multivariate (pre)wavelets," Constr. Approx. 9(2), 1993.

3. I. Khalidov and M. Unser, "From differential equations to the construction of wavelet-like bases," IEEE Trans. Sig. Proc., 2005. To be published.

4. N. Dyn, D. Levin, and A. Luzzatto, "Exponentials reproducing subdivision schemes," Found. Comput. Math. 3(2), 2003.

5. G. Deslauriers and S. Dubuc, "Symmetric iterative interpolation processes," Constr. Approx. 5, 1989.

6. A. Cohen, I. Daubechies, and J.-C. Feauveau, "Biorthogonal bases of compactly supported wavelets," Comm. Pure Appl. Math. 45(5), 1992.

7. M. Unser and T. Blu, "Cardinal exponential splines: Part I - Theory and filtering algorithms," IEEE Trans. Sig. Proc. 51(2), 2004.

8. A. Antonini, M. Barlaud, P. Mathieu, and I. Daubechies, "Image coding using wavelet transform," IEEE Trans. on Image Processing 1(2), pp. 205-220, 1992. 